\title{
Untersuchungen zum Einfluss der Holzfeuchte auf ausgewählte Eigenschaften von Spanplatten und MDF
}

\author{
Journal Article \\ Author(s): \\ Pavlekovics, András; Niemz, Peter; Sonderegger, Walter; Molnar, Sandor \\ Publication date: \\ 2008 \\ Permanent link: \\ https://doi.org/10.3929/ethz-b-000106205
}

Rights / license:

In Copyright - Non-Commercial Use Permitted

Originally published in:

Holz als Roh- und Werkstoff 66(2), https://doi.org/10.1007/s00107-007-0199-z 


\title{
Untersuchungen zum Einfluss der Holzfeuchte auf ausgewählte Eigenschaften von Spanplatten und MDF
}

\author{
Andràs Pavlekovics $\cdot$ Peter Niemz $\cdot$ Walter Sonderegger $\cdot$ Sandor Molnar
}

Published online: 25 September 2007

(C) Springer-Verlag 2007

\begin{abstract}
Zusammenfassung An industriell gefertigten Spanplatten und MDF wurde der Einfluss der Holzfeuchte auf den EModul bei Biegebelastung, die Biegefestigkeit sowie die Schubmoduln $\mathrm{G}_{z x}$ und $\mathrm{G}_{z y}$ ermittelt. Die Gleichgewichtsfeuchte und die differentielle Dickenquellung waren bei MDF niedriger als bei Spanplatten, was sich mit den Erkenntnissen bisheriger Arbeiten deckt. Ebenso sinken die Zugfestigkeit, der E-Modul, der Schubmodul und die Biegefestigkeit bei Erhöhung der Holzfeuchte. Die Poissonsche Konstante $\mu_{12}$ sinkt mit zunehmender Holzfeuchte.
\end{abstract}

\section{Studies on the influence of wood moisture content} on selected properties of particle boards and MDF

\begin{abstract}
On industrially produced particle boards and MDF, the influence of wood moisture on bending MOE, bending strength and shear modulus $\mathrm{G}_{z x}$ and $\mathrm{G}_{z y}$ was determined. The equilibrium moisture content and the differential swelling of thickness proved to be smaller for MDF than for particle boards. Tensile strength and tensile MOE, shear modulus and bending strength decrease likewise with increasing wood moisture content. Furthermore the Poisson ratio $\mu_{12}$ decreases with increasing wood moisture content.
\end{abstract}

A. Pavlekovics $\cdot$ S. Molnar

Fakultät für Holzwissenschaften,

Westungarische Universität Sopron,

Sopron, Ungarn

P. Niemz (®) · W. Sonderegger

Institut für Baustoffe, Arbeitsgruppe Holzphysik, ETH Zürich,

Schafmattstrasse 6,

8093 Zürich, Schweiz

e-mail:niemzp@ethz.ch

\section{Einleitung}

Spanplatten, MDF und OSB gewinnen im Bauwesen im konstruktiven Bereich zunehmend an Bedeutung. Für rechnerische Lösungen mit Finite Elemente Programmen sind komplexe Kenntnisse der Materialkennwerte erforderlich. So müssen E- und Schubmoduli aber auch Poissonsche Konstanten bekannt sein. Ebenso sind Kenntnisse des Einflusses der Holzfeuchte erforderlich. Bisher gibt es vergleichsweise wenige Arbeiten zu dieser Thematik. Dazu wurden in der vorliegenden Arbeit Messungen an industriell gefertigten Platten durchgeführt.

\section{Erkenntnisstand}

An Holzpartikelwerkstoffen werden standardmässig überwiegend die E-Moduli bei Biegung, die Biegefestigkeit und die Querzugfestigkeit geprüft. Zug- und Druckfestigkeit in Plattenebene, sowie Schubmodul und insbesondere Poissonsche Konstanten zählen zu den wenig bis kaum geprüften Eigenschaften. Eine vergleichsweise komplette Übersicht zu den üblicherweise geprüften mechanischen Eigenschaften geben Deppe und Ernst (2000).

Die umfangreichsten Untersuchungen zur Bestimmung der Poissonschen Konstanten von Spanplatten und Sperrholz führte Albers (1970) durch. Dabei wurde allerdings nur im Normalklima bei $20^{\circ} \mathrm{C} / 65 \%$ rel. Luftfeuchte geprüft. Der Einfluss der Holzfeuchte wurde bisher nicht untersucht.

Schulte (1997) untersuchte technologische Einflussgrößen auf die verschiedenen Eigenschaften von Holzwerkstoffen, so auch den Schubmodul, und deren Korrelation untereinander.

Auch zum Einfluss der Holzfeuchte auf den E-Modul, die Biegefestigkeit und den Schubmodul von Holzpartikel- 
werkstoffen liegen vergleichsweise wenige Untersuchungen vor. Halligan und Schniewind (1974) ermittelten einen nahezu linearen Abfall von E-Modul und Zugfestigkeit senkrecht zur Plattenebene von Spanplatten mit zunehmender Holzfeuchte im praktisch relevanten Bereich zwischen $8 \%-18 \%$ Holzfeuchte.

Generell variieren in der Literatur die Angaben zum Schubmodul sehr stark. In älteren Arbeiten wird meist ein Schubmodul bei Beanspruchung senkrecht zur Plattenebene $\left(\mathrm{G}_{z x}, \mathrm{G}_{z y}\right)$ von ca. $200 \mathrm{~N} / \mathrm{mm}^{2}$ angegeben (z.B. Deppe und Ernst 2000). Teilweise werden aber heute in Firmenschriften deutlich tiefere Werte um etwa $80-100 \mathrm{~N} / \mathrm{mm}^{2}$ angegeben. Im Rahmen der vorliegenden Arbeit wurde der Einfluss der Holzfeuchte auf den E-Modul bei Biegung, die Biegefestigkeit, den E-Modul bei Zug parallel zur Plattenebene, die Zugfestigkeit in Plattenebene, den Schubmodul $\mathrm{G}_{z x}$ und $\mathrm{G}_{z y}$ und exemplarisch eine der 6 Poissonschen Konstanten $\left(\mu_{12}\right)$ bestimmt.

\section{Versuchsmaterial und -methodik}

\subsection{Versuchsmaterial}

Als Versuchsmaterial wurden industriell gefertigte, mit Harnstoffharz verklebte Spanplatten (Rohdichte $670 \mathrm{~kg} / \mathrm{m}^{3}$ ) und MDF (Rohdichte $720 \mathrm{~kg} / \mathrm{m}^{3}$ ) verwendet.

\subsection{Versuchsmethodik}

\subsubsection{Versuchsübersicht}

Es wurden

- Zugstäbe (Abb. 1) in Anlehnung an DIN 52377 (1978), Probendicke entspricht Plattendicke, und

- Biegestäbe $400 \mathrm{~mm}$ (Länge) $\times 50 \mathrm{~mm}$ (Breite) $\times$ Plattendicke

gefertigt.

Je Variante wurden 20 Proben parallel und 20 Proben senkrecht zur Herstellungsrichtung entnommen und geprüft.
Die Prüfung erfolgte zunächst getrennt in und senkrecht zur Herstellungsrichtung, danach wurden die Werte als Mittelwert zusammengefasst.

Die Proben wurden in einer Klimakammer bzw. in klimatisierten Räumen bei jeweils $20^{\circ} \mathrm{C}$ und variabler relativer Luftfeuchte bis zum Erreichen der Gleichgewichtsfeuchte klimatisiert. Dabei wurde die rel. Luftfeuchte von $35 \%$ bis zu 93\% variiert. Auf die Prüfung des darrtrockenen Zustandes wurde verzichtet, da bei Partikelwerkstoffen durch die Darrtrocknung eine irreversible Schädigung der Partikelbindungen eintritt.

Nach Versuchsabschluss wurde mittels Darrmethode der Feuchtegehalt ermittelt.

\subsubsection{Bestimmung des E-Moduls und des Schubmoduls bei Dreipunktbiegung}

In Anlehnung an DIN EN 408 (2004) wurde durch Dreipunkt-Biegeprüfung mit variabler Stützweite der E-Modul bei reiner Biegung und der Schubmodul berechnet. Das der Norm zugrunde liegende Verfahren wurde auch von Albers (1970) für die Bestimmung des Schubmoduls von Spanplatten angewandt. Dabei wird der Effekt genutzt, dass der E-Modul bei Dreipunktbiegung sich aus den Komponenten der reinen Biegung und der Schubverformung zusammensetzt. Mit steigendem Verhältnis Stützweite/Dicke sinkt der Einfluss der Schubverformung, der E-Modul bei Dreipunktbiegung $\mathrm{E}_{\mathrm{m} \text {,app }}$ steigt. Daher lassen sich bei Variation des Verhältnisses Stützweite/Dicke der E-Modul bei reiner Biegung und der Schubmodul aus den Koeffizienten $k_{1}$ und $k_{2}$ einer Geradengleichung bestimmen.

Es gilt:

$$
1 / \mathrm{E}_{m, \text { app }}=f(h / l)^{2}
$$

Dabei sind:

$\mathrm{E}_{\mathrm{m}, \text { app }}$ - scheinbarer Biege-E-Modul

h - Probenhöhe

1 - Stützweite

Aus den Konstanten der Geradengleichung können dann der Schubmodul $\mathrm{G}\left(\mathrm{G}_{z x}, \mathrm{G}_{z y}\right)$ und der E-Modul bei rei-
Abb. 1 Zugstab (Dicke entspricht der Plattendicke)

Fig. 1 Tensile sample

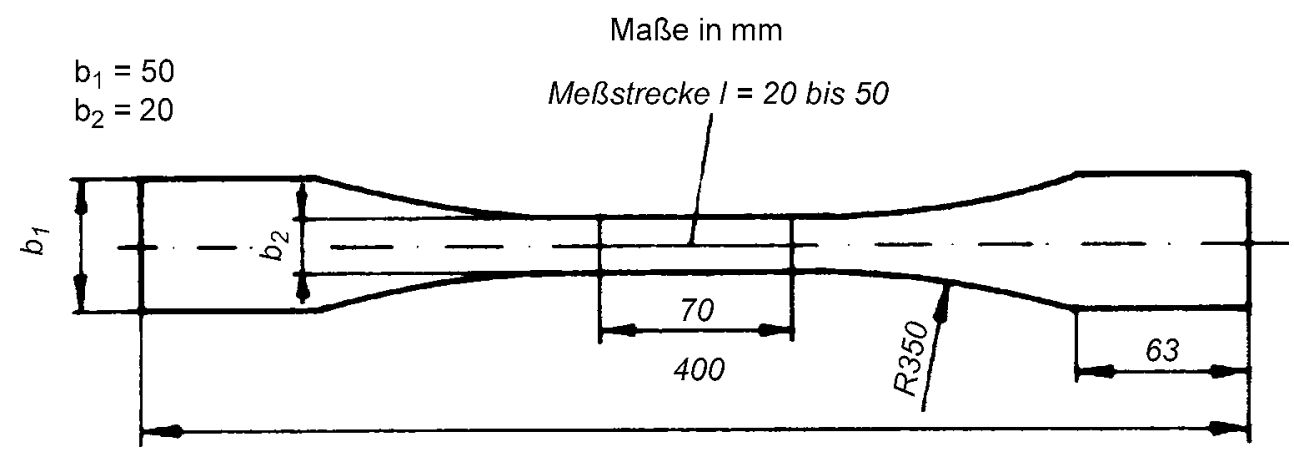


ner Biegung $\mathrm{E}_{\mathrm{m}}$ bestimmt werden. Berechnet wurde der Mittelwert beider Kennwerte unter Vernachlässigung der Differenzen beider Richtungen, d.h. bei Annahme einer Flächenisotropie.

$$
\begin{aligned}
& G=\frac{1,2}{k_{1}}\left[\mathrm{~N} / \mathrm{mm}^{2}\right] \\
& E_{\mathrm{m}}=1 / k_{2}
\end{aligned}
$$

\section{Dabei sind:}

$k_{1}$ - die Steigung der Ausgleichsgeraden nach G1. 1

$\mathrm{G}$ - der Schubmodul und

$\mathrm{E}_{\mathrm{m}}$ - der E-Modul bei reiner Biegung

Das detaillierte Vorgehen ist in DIN EN 408 (2004) beschrieben.

\subsubsection{Bestimmung der E-Moduln,} der Poissonschen Konstanten und der Zugfestigkeit

An den in Anlehnung an DIN 52377 (1978) gefertigten Zugstäben wurde der E-Modul bei Zugbelastung in Plattenebene ermittelt. Die Prüfung erfolgte innerhalb des proportionalen Bereiches des Spannungs-Dehnungs-Diagramms. Dabei wurde mit dem Videosystem VIC 2D (Video Image Correlation) über auf die Probenoberfläche aufgebrachte Speckle-Muster die Verzerrung der Probe und daraus die Dehnung bestimmt. Als Untergrund für das Speckle-Muster wurde weisses Gipspulver aufgebracht, auf dieses wurde

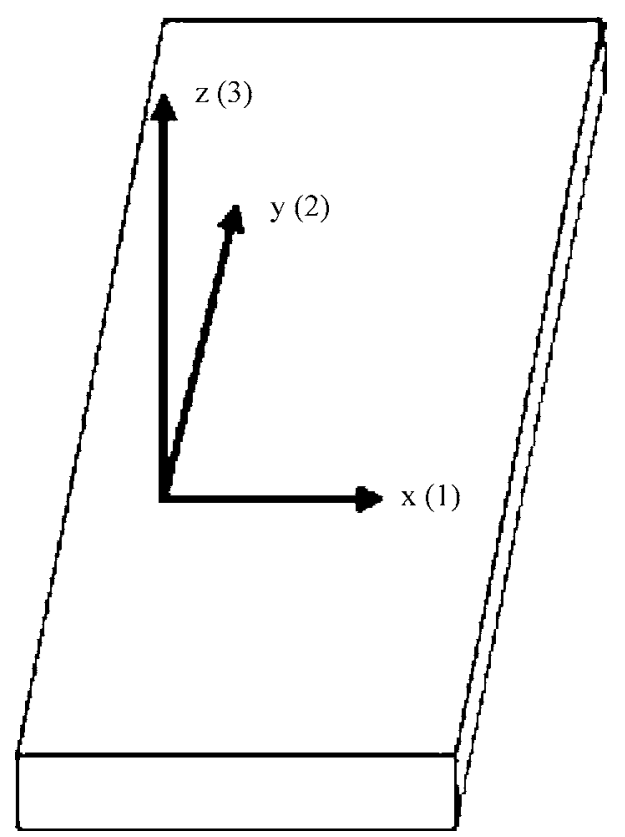

Abb. 2 Koordinatensystem zur Bestimmung der Poissonschen Konstante. y ... Herstellungsrichtung der Platten; x ... senkrecht zur Herstellungsrichtung der Platte; z ... senkrecht zur Plattenebene

Fig. 2 Coordinate system for the determination of the Poisson ratio dann schwarzer Tonerpuder mittels eines Siebs gleichmäßig verteilt. Diese Speckle dienten dann als Basis für die Kreuzkorrelation zur Ermittlung der Verzerrung der Proben unter Last.

Aus den Dehnungen in und senkrecht zur Belastungsrichtung wurde dann die Poissonsche Konstante $\mu_{12}$ gemäss Abb. 2 bei Belastung in Plattenebene bestimmt. Dabei ist 1 die Richtung der Belastung (in Fertigungsrichtung), 2 die Richtung der Kontraktion (senkrecht zur Fertigungsrichtung).

Die Poissonsche Konstante berechnet sich dann zu:

$\mu=-\frac{\varepsilon_{q}}{\varepsilon_{l}}$

$\mu$ - Poissonsche Konstante

$\varepsilon_{l}$ - Dehnung in Belastungsrichtung

$\varepsilon_{q}-$ Dehnung senkrecht zur Belastungsrichtung

\section{Versuchsergebnisse}

\subsection{Sorptionsverhalten und Dickenquellung}

MDF und Spanplatten unterschieden sich deutlich im Sorptionsverhalten (Tabelle 1). Bei MDF ist die Gleichgewichtsfeuchte niedriger als bei Spanplatten, was möglicherweise auf die hydrothermische Vorbehandlung beim Defibrieren zurückzuführen ist. Dies wirkt sich auch auf die Quellung aus (siehe z.B. (Krug und Tobisch 2006, Xu und Suchsland 1991)). Die differentielle Dickenquellung (linearisiert zwischen $35 \%$ und $93 \%$ rel. Luftfeuchte und bezogen auf die Dicke bei $35 \%$ rel. Luftfeuchte) wurde für MDF

Tabelle 1 Einfluss der Holzfeuchte auf E-modul, Biegefestigkeit und Schubmodul von MDF und Spanplatte. $\mathrm{E}_{m}=\mathrm{E}-$ Modul bei reiner Biegung; $\mathrm{E}_{m}$, app $=\mathrm{E}-$ Modul bei Dreipunktbiegung; $\mathrm{G}=$ Schubmodul (Mittelwert aus $\mathrm{G}_{z x}$ und $\mathrm{G}_{z y}$ )

Table 1 Influence of wood moisture content on MOE, bending strength and shear modulus of $\mathrm{MDF}$ and particleboards. $\mathrm{E}_{m}=\mathrm{E}$ modulus for absolute bending; $\mathrm{E}_{m \text {, app }}=\mathrm{E}$-modulus in 3-point bending tests; $\mathrm{G}=$ shear modulus (mean value of $\mathrm{G}_{z x}$ and $\mathrm{G}_{z y}$ )

\begin{tabular}{rrrrrr}
\hline $\begin{array}{c}\text { Holzfeuchte } \\
{[\%]}\end{array}$ & $\begin{array}{c}\text { Dicke } \\
{[\mathrm{mm}]}\end{array}$ & $\begin{array}{c}\mathrm{E}_{\mathrm{m}} \\
{\left[\mathrm{N} / \mathrm{mm}^{2}\right]}\end{array}$ & $\begin{array}{c}\mathrm{E}_{\mathrm{m}, \text { app }} \\
{\left[\mathrm{N} / \mathrm{mm}^{2}\right]}\end{array}$ & $\begin{array}{c}\text { Biegefestigkeit } \\
{\left[\mathrm{N} / \mathrm{mm}^{2}\right]}\end{array}$ & $\begin{array}{c}\mathrm{G} \\
{\left[\mathrm{N} / \mathrm{mm}^{2}\right]}\end{array}$ \\
\hline \multicolumn{5}{c}{$M D F$} \\
6,1 & 15,87 & 5147 & 4294 & 42,5 & 115,9 \\
6,9 & 15,93 & 4837 & 3911 & 37,0 & 108,3 \\
10,0 & 16,32 & 3805 & 3083 & 30,9 & 78,5 \\
10,8 & 16,42 & 3511 & 2793 & 25,5 & 60,7 \\
16,8 & 17,31 & 2424 & 1471 & 15,4 & 26,3 \\
\multicolumn{5}{c}{ Spanplatte } \\
6,9 & 15,99 & 2715 & 2423 & 13,4 & 64,6 \\
8,9 & 16,21 & 2458 & 2203 & 12,3 & 59,6 \\
13,1 & 16,80 & 2100 & 1773 & 9,5 & 37,1 \\
13,5 & 16,87 & 2026 & 1638 & 8,9 & 34,2 \\
18,2 & 17,78 & 1731 & 1189 & 6,7 & 23,8 \\
\hline
\end{tabular}


mit $0,85 \% / \%$ Feuchteänderung und für die Spanplatten mit $0,99 \% / \%$ Feuchteänderung ermittelt. Sie liegt etwa in dem von Sonderegger und Niemz (2006) angegebenen Bereich. Dabei ist natürlich eine Streuung der Kennwerte in Variation der Rohdichte und der Plattenstruktur (Partikellänge, Klebstoffanteil) vorhanden, dies zeigt sich auch an der Variation der von Sonderegger und Niemz (2006) ermittelten Kennwerte für die Quellung.

\subsection{Biege-Elastizitätsmodul- und Schubmodul, \\ Biegefestigkeit}

Tabelle 1 sowie Abbildungen 3-7 zeigen die Ergebnisse. Es ist ein geringer Einfluss der Entnahmerichtung (in und senk- recht zur Herstellungsrichtung) der Platten vorhanden (ca. $10 \%$ höhere Werte in Fertigungsrichtung). Bei der Auswertung wurden die Ergebnisse beider Orientierungsrichtungen zusammengefasst, um den Probenumfang zu erhöhen (40 Proben je Variante). Die leicht höheren Werte in Herstellungsrichtung sind auf einen gewissen Orientierungseffekt der Partikel bei der Fertigung zurückzuführen.

Mit zunehmender Holzfeuchte sinken der E-Modul, der Schubmodul und die Biegefestigkeit deutlich ab (Abb. 3, 4, 6 und 7). Der E-Modul bei Dreipunktbelastung und einem Verhältnis Stützweite/Dicke $=20$ liegt um ca. 15\%-20\% unter dem bei reiner Biegung ermittelten. Abbildung 5 zeigt den Einfluss des Verhältnisses Stützweite/Probendicke bei Dreipunktbiegung auf den Biege-E-Modul für Spanlatten
Abb. 3 Einfluss der Holzfeuchte auf den E-Modul von MDF bei reiner Biegung und Dreipunktbiegung (Verhältnis

Stützweite/Dicke $=20$ )

Fig. 3 Influence of moisture content on MOE of MDF for absolute bending and in 3-point bending tests, span width: $320 \mathrm{~mm}$ (span width/thickness $=20$ )

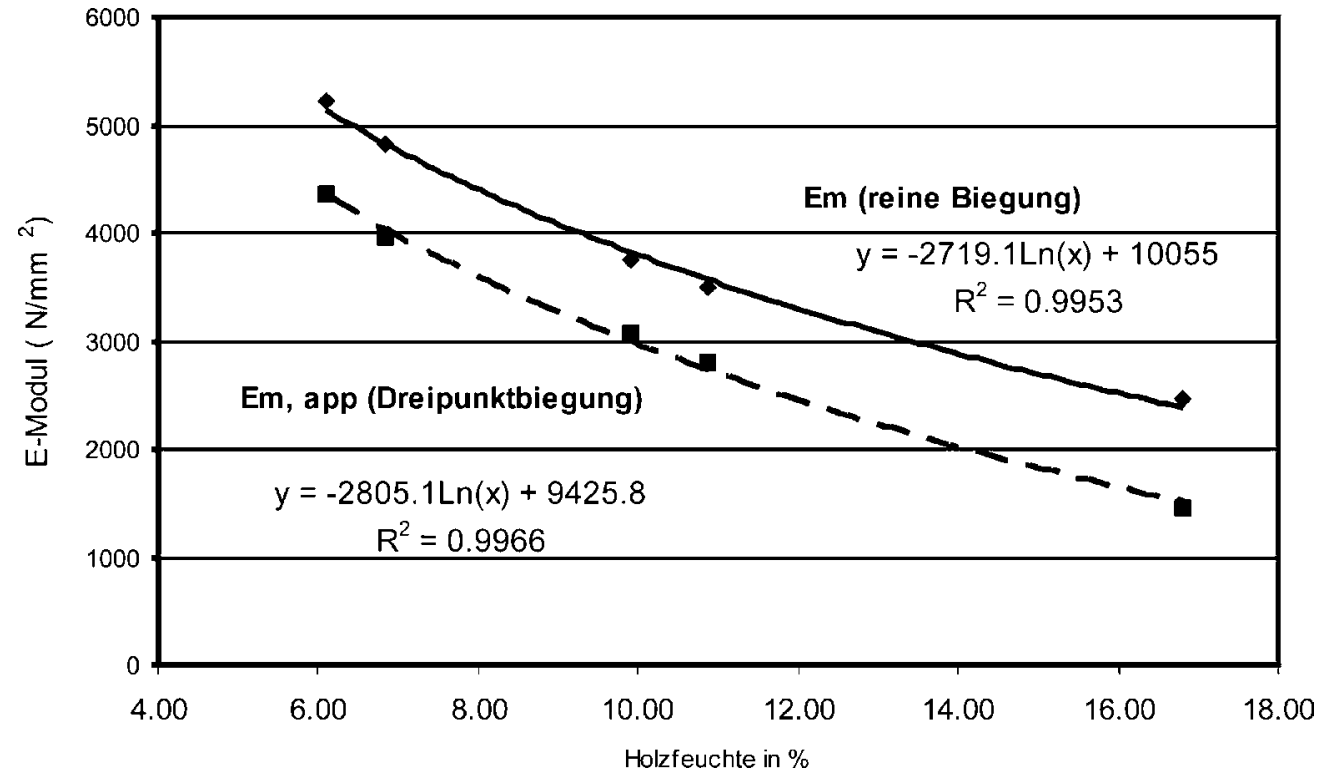

Abb. 4 Einfluss der Holzfeuchte auf den E-Modul von Spanplatten bei reiner Biegung und Dreipunktbiegung (Verhältnis

Stützweite/Dicke $=20$ )

Fig. 4 Influence of moisture content on MOE of particleboard for absolute bending and in 3-point bending tests, span width: $320 \mathrm{~mm}$ ( span width/thickness $=20$ )

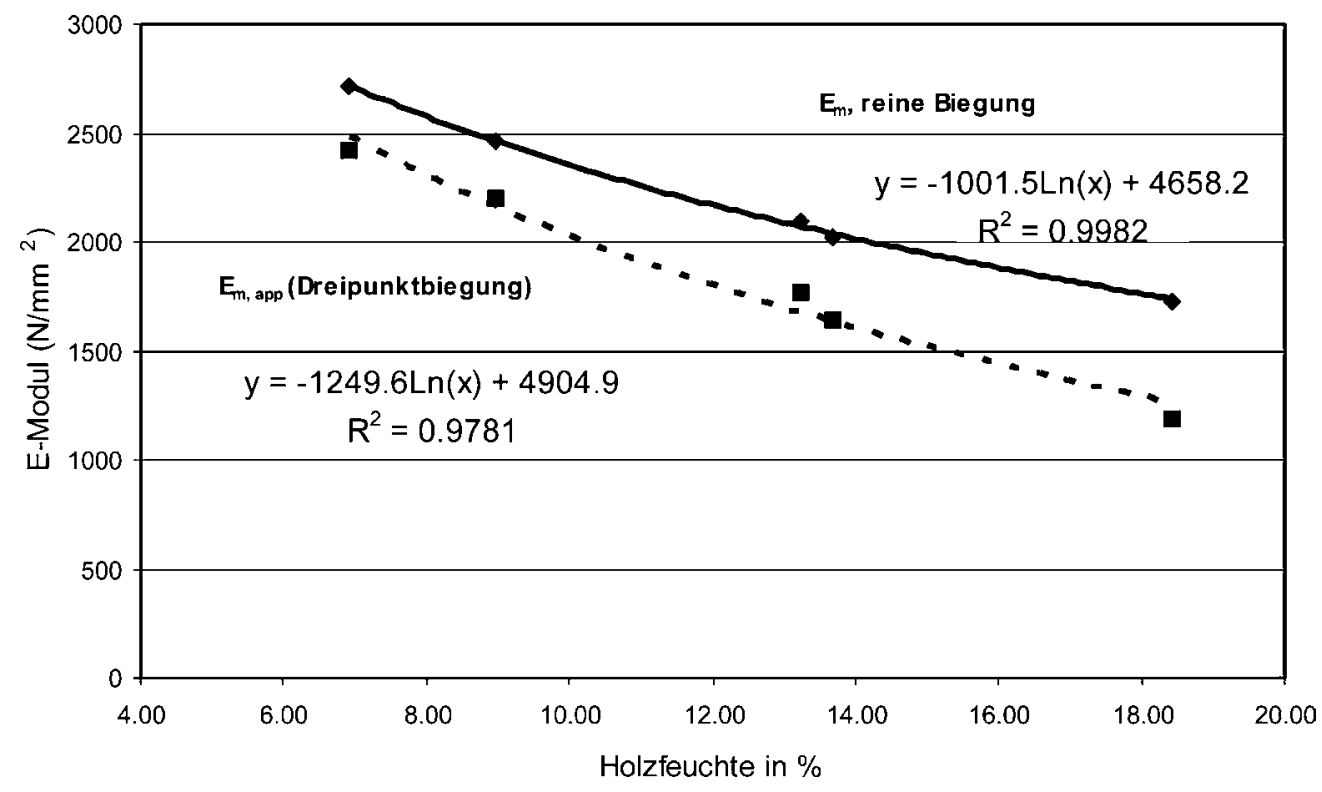


Abb. 5 Einfluss des

Verhältnisses Stützweite (1)/Dicke

(a) auf den E-Modul $\mathrm{E}_{m \text {,app }}$ bei

Dreipunkt-Biegung von

Spanplatte und MDF bei

Lagerung im Normalklima bei

$20{ }^{\circ} \mathrm{C} / 65 \%$ rel. Luftfeuchte

Fig. 5 Influence of the relation of span width (1) to thickness (a) on MOE $\mathrm{E}_{m \text {,app }}$ in 3-point bending tests for particle boards and MDF at $20{ }^{\circ} \mathrm{C}$ and $65 \%$ rel. air moisture

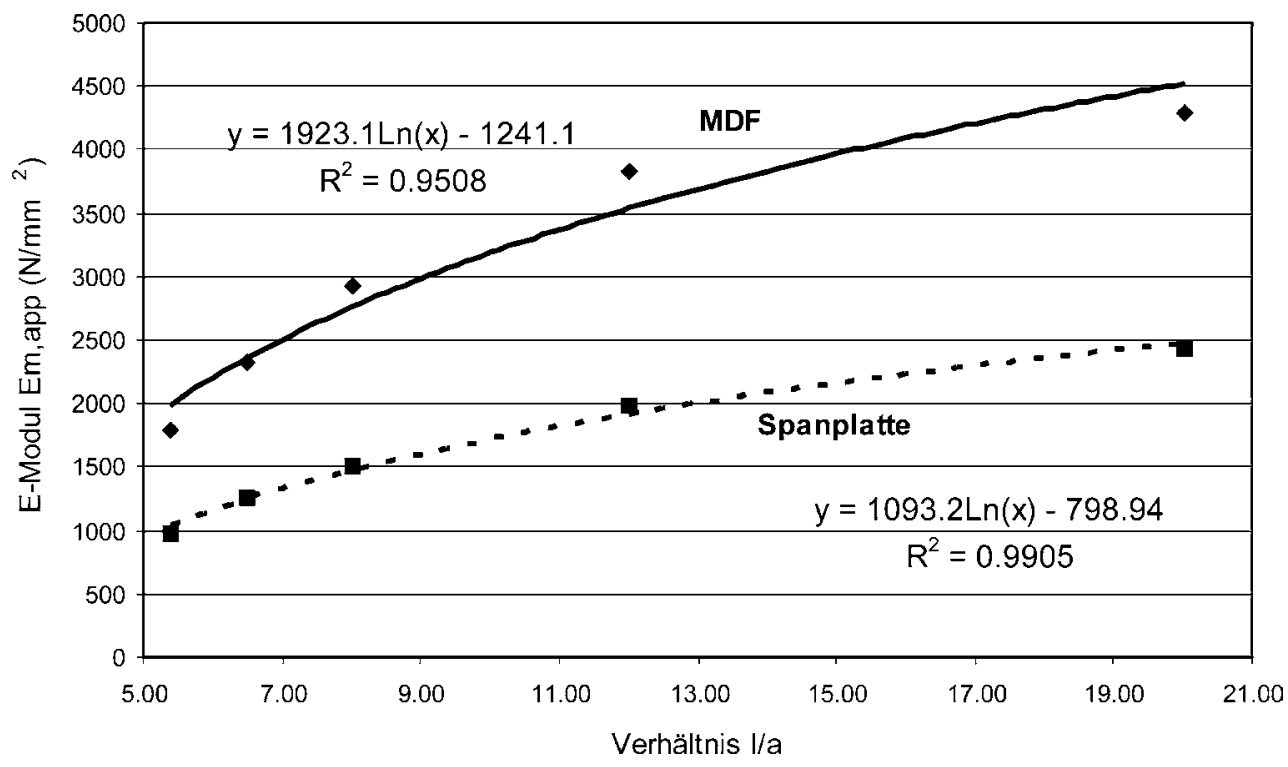

Abb. 6 Einfluss der Holzfeuchte auf die Biegefestigkeit von MDF und Spanplatte Fig. 6 Influence of wood moisture on bending strength for MDF and particle board

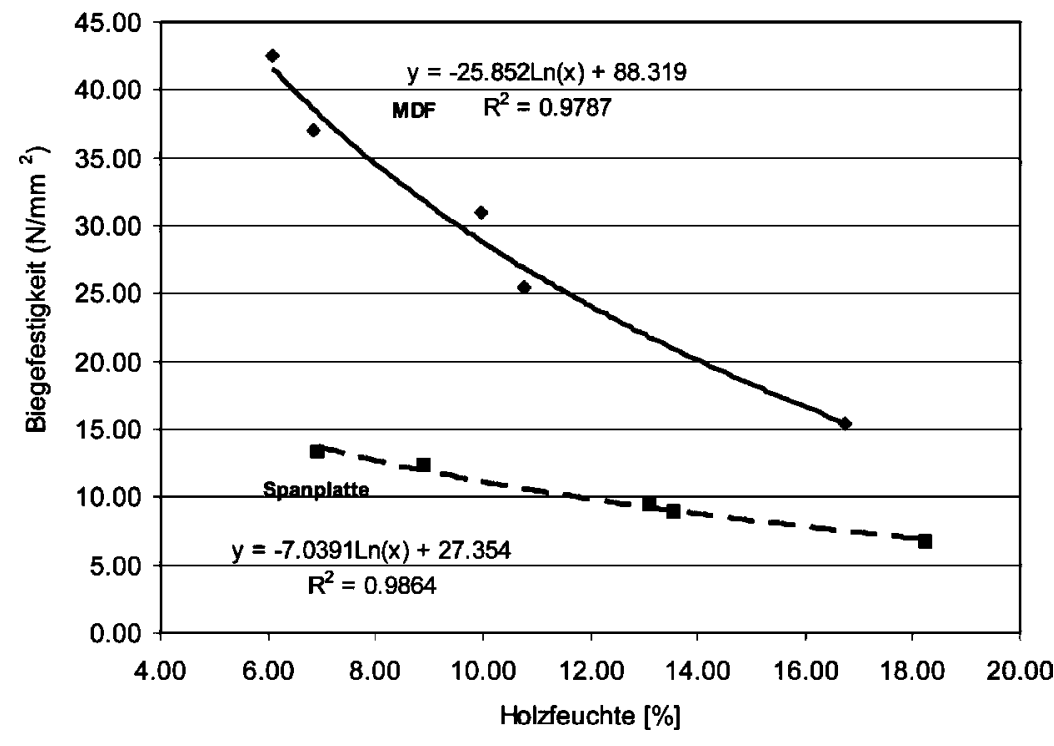

und MDF im Normalklima. Mit zunehmendem Verhältnis Stützweite/Probendicke steigt der E-Modul bei Dreipunktbiegung deutlich an.

Für den Einfluss der Holzfeuchte ergeben sich folgende Werte für die prozentuale Eigenschaftsänderung in $\% / \%$ Holzfeuchteänderung:

Für MDF

$\mathrm{E}_{\mathrm{m}} \quad=254 \mathrm{~N} / \mathrm{mm}^{2} / \%$ Feuchteänderung

$\mathrm{E}_{\mathrm{m} \text {,app }} \quad=264 \mathrm{~N} / \mathrm{mm}^{2} / \%$ Feuchteänderung

Biegefestigkeit $=2,54 \mathrm{~N} / \mathrm{mm}^{2} / \%$ Feuchteänderung

$\mathrm{G} \quad=8,4 \mathrm{~N} / \mathrm{mm}^{2} / \%$ Feuchteänderung

\section{Für Spanplatten}

$\mathrm{E}_{\mathrm{m}} \quad=87,1 \mathrm{~N} / \mathrm{mm}^{2} / \%$ Feuchteänderung

$\mathrm{E}_{\mathrm{m} \text {,app }} \quad=109,2 \mathrm{~N} / \mathrm{mm}^{2} / \%$ Feuchteänderung
Biegefestigkeit $=0,59 \mathrm{~N} / \mathrm{mm}^{2} / \%$ Feuchteänderung

$\mathrm{G} \quad=3,6 \mathrm{~N} / \mathrm{mm}^{2} / \%$ Feuchteänderung

Der Eigenschaftsverlust mit zunehmender Holzfeuchte ist im untersuchten Bereich nahezu linear.

\subsection{Zug-Elastizitätsmodul und Poissonsche Konstanten}

Tabelle 2 und die Abbildungen 8 und 9 zeigen den Einfluss der Holzfeuchte auf den E-Modul bei Zugbelastung und die Zugfestigkeit. Generell ist festzustellen, dass (wie bekannt) bei Holzpartikelwerkstoffen die Biegefestigkeit deutlich über der Zugfestigkeit liegt (vgl. Tabellen 1 und 2). Dieses Verhältnis gilt auch für den E-Modul bei Biegung. Auch hier liegen die bei Biegung 


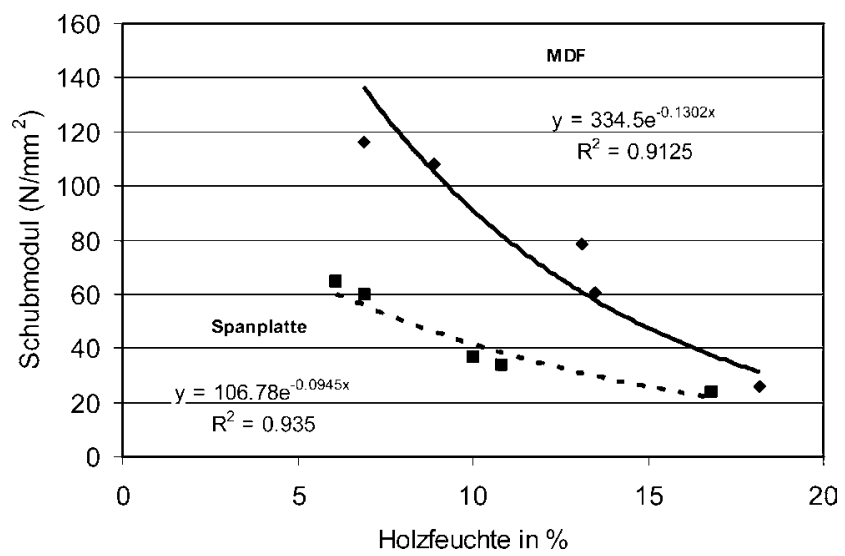

Abb. 7 Einfluss der Holzfeuchte auf den Schubmodul von Spanplatte und MDF

Fig. 7 Influence of wood moisture on shear modulus for particle board

Tabelle 2 Einfluss der Holzfeuchte auf den E-Modul bei Zug, die Zugfestigkeit und die Poissonsche Konstante $\mu_{12}$ für Spanplatten und MDF

Table 2 Influence of wood moisture content on MOE for tension, tensile strength and Poisson ratio $\mu_{12}$ of particleboard and MDF

\begin{tabular}{rcccc}
\hline $\begin{array}{c}\text { Holzfeuchte } \\
{[\%]}\end{array}$ & $\begin{array}{c}\text { E-Modul } \\
{\left[\mathrm{N} / \mathrm{mm}^{2}\right]}\end{array}$ & $\begin{array}{c}\text { Zugfestigkeit } \\
{\left[\mathrm{N} / \mathrm{mm}^{2}\right]}\end{array}$ & $\begin{array}{c}\text { Poissonzahl } \\
{[-]}\end{array}$ & $\begin{array}{c}\text { Stand.abw. } \\
\text { Poisson }\end{array}$ \\
\hline \multicolumn{5}{c}{$M D F$} \\
6,1 & 3639 & 22,96 & 0,48 & 0,11 \\
7,7 & 2689 & 20,40 & 0,37 & 0,02 \\
11,0 & 2260 & 15,65 & 0,35 & 0,06 \\
16,6 & 1407 & 10,71 & 0,31 & 0,037 \\
& & Spanplatte & \\
6,8 & 1917 & 7,51 & 0,31 & 0,047 \\
9,5 & 1822 & 7,15 & 0,26 & 0,053 \\
13,5 & 1586 & 5,21 & 0,24 & 0,049 \\
18,2 & 1073 & 3,28 & 0,23 & 0,039 \\
\hline
\end{tabular}

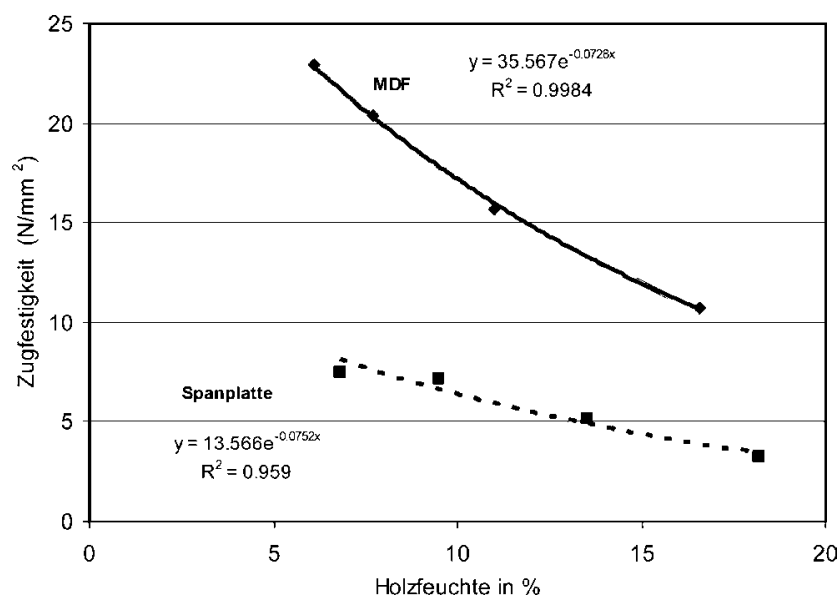

Abb. 8 Einfluss der Holzfeuchte auf die Zugfestigkeit von MDF und Spanplatte

Fig. 8 Influence of wood moisture on tensile strength for MDF and particle board

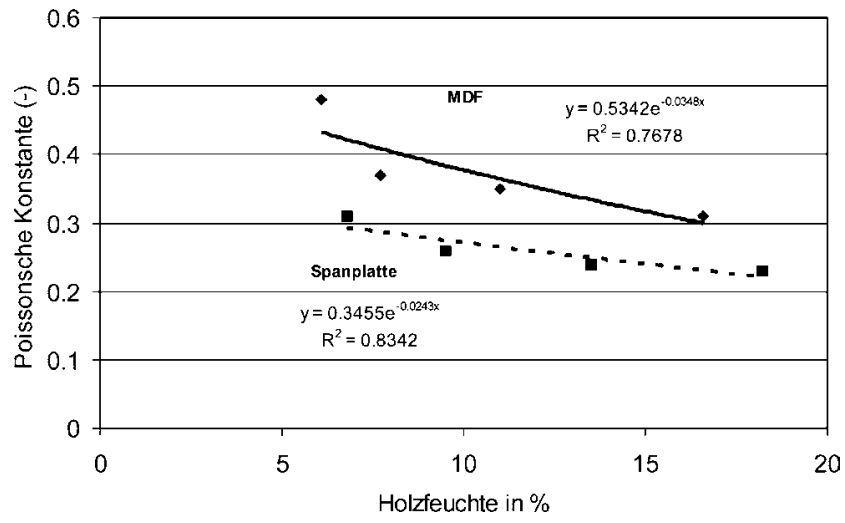

Abb.9 Einfluss der Holzfeuchte auf die Poissonsche Konstante $\mu_{12}$ von MDF und Spanplatte

Fig. 9 Influence of wood moisture on Poisson ratio for MDF and particle board

bestimmten E-Moduli deutlich über den bei Zug ermittelten. Die Poissonsche Konstante sinkt mit zunehmender Holzfeuchte ab, dies steht im Gegensatz zu den Messungen von Neuhaus (1981), der bei Fichte einen Anstieg ermittelte.

Es ergeben sich folgende Werte für die prozentuale Eigenschaftsänderung in $\% / \%$ Materialfeuchteänderung.

Für MDF
E-Modul
$=212,6 \mathrm{~N} / \mathrm{mm}^{2} / \%$
Feuchteänderung
Zugfestigkeit
$=1,16 \mathrm{~N} / \mathrm{mm}^{2} / \%$
Feuchteänderung

Poissonsche Konstante $=0,016 / \%$ Feuchteänderung

für Spanplatte

$\begin{array}{ll}\text { E-Modul } & =74 \mathrm{~N} / \mathrm{mm}^{2} / \% \text { Feuchteänderung } \\ \text { Zugfestigkeit } & =0,37 \mathrm{~N} / \mathrm{mm}^{2} / \% \\ & \text { Feuchteänderung } \\ \text { Poissonsche Konstante } & =0,0070 / \% \text { Feuchteänderung }\end{array}$

\section{Zusammenfassung und Schlussfolgerungen}

An industriell gefertigten Spanplatten und MDF wurden bei Variation der Holzfeuchte der E-Modul bei Zugbelastung in Plattenebene, die Poissonsche Konstante $\mu_{12}$, die Zugfestigkeit sowie der E-Modul bei reiner Biegung, bei Querkraftbiegung und der Schubmodul $\mathrm{G}_{z x}, \mathrm{G}_{z y}$ sowie die Poissonsche Konstante bestimmt. Bei Erhöhung der Holzfeuchte sinken im untersuchten Feuchtebereich von etwa 6\%-18\% die E- und G-Module sowie die Poissonsche Konstante nahezu linear ab. Die Gleichgewichtsfeuchte der MDF ist leicht niedriger als die der Spanplatten.

Es wurden unter Annahme eines im untersuchten Bereich linearen Einflusses der Holzfeuchte Kennwerte für eine 
prozentuale Änderung der Holzeigenschaften je \% Holzfeuchteänderung bestimmt.

Danksagung Die Bearbeitung erfolgte im Rahmen eines von der ETH Zürich finanzierten Stipendiums von Herrn Pavlekovics am Institut für Baustoffe der ETH

\section{Literatur}

Albers K (1970) Querdehnungs- und Gleitzahlen sowie Schub- und Scherfestigkeiten von Holzwerkstoffen. Dissertation, Universität Hamburg

DIN EN 408 (2004) Holzbauwerke. Bauholz für tragende Zwecke und Brettschichtholz. Beuth Verlag, Berlin

DIN 52377 (1978) Prüfung von Sperrholz. Bestimmung des Zug-Elastizitätsmoduls und der Zugfestigkeit. Beuth Verlag, Berlin
Deppe HJ, Ernst K (2000) Taschenbuch der Spanplattentechnik. 4. Auflage. DRW-Verlag, Stuttgart

Halligan AF, Schniewind AP (1974) Prediction of particleboard mechanical properties of various moisture content. Wood Sci Technol 8:68-78

Krug D, Tobisch S (2006) Holzfaserplattenaufschluss bei hohen Temperaturen und Drücken - Einfluss auf die Eigenschaften von MDF. Forstwiss. Tagung, Tharandt-Dresden 20.-22.9.2006

Neuhaus HF (1981) Elastizitätszahlen von Fichtenholz. Diss. Ruhruniversität Bochum

Schulte M (1997) Zerstörungsfreie Prüfung elastomechanischer Eigenschaften von Holzwerkstoffplatten durch Auswertung des Eigenschwingungsverhaltens und Vergleich mit zerstörenden Prüfmethoden. Diss. Universität Hamburg

Sonderegger W, Niemz P (2006) Untersuchungen zur Quellung und Wärmedehnung von Faser-, Span- und Sperrholzplatten. Holz Roh- Werkst 64:11-20

Xu H, Suchsland O (1991) The expansion potential: A new evaluator of expansion behavior of wood composites. For Prod J 41:39-42 